\title{
The origin of fluorine: abundances in AGB carbon stars revisited (Corrigendum)
}

\author{
C. Abia ${ }^{1}$, K. Cunha ${ }^{2}$, S. Cristallo ${ }^{3,4}$, and P. de Laverny ${ }^{5}$
}

1 Dpto. Física Teórica y del Cosmos, Universidad de Granada, 18071 Granada, Spain e-mail: cabia@ugr.es

2 Observatório Nacional, Rua General José Cristino, 77, 20921-400 São Critovão, Rio de Janeiro, RJ, Brazil

3 INAF, Osservatorio Astronomico di Collurania, 64100 Teramo, Italy

4 INFN Sezione Napoli, Napoli, Italy

5 Laboratoire Lagrange, Université Côte d'Azur, Observatoire de la Côte d'Azur, CNRS, CS 34229, 06304 Nice Cedex 4, France

A\&A 581, A88 (2015), DOI: 10.1051/0004-6361/201526586

Key words. stars: AGB and post-AGB - stars: abundances - nuclear reactions, nucleosynthesis, abundances - errata, addenda

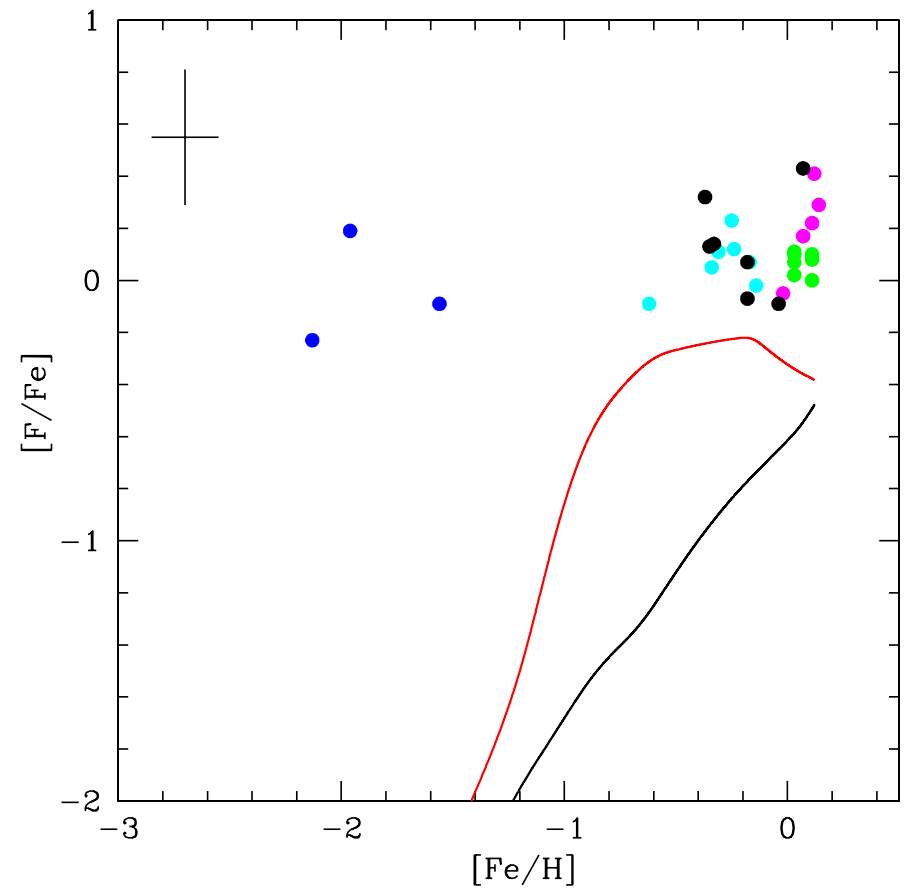

Fig. 4. $[\mathrm{F} / \mathrm{Fe}]$ vs. $[\mathrm{Fe} / \mathrm{H}]$ observed in Galactic field dwarfs and giants and open cluster stars compared with the predicted evolution from our GCE model for the solar neighbourhood including only fluorine production from AGB stars. Black line: yields from Cristallo et al. (2015); red line: yields from Karakas (2010). The observed ratios (circles) are from Recio-Blanco et al. (2012; black), Li et al. (2013; blue), Maiorca et al. (2014; green), Jönsson et al. (2014; cyan), and Nault \& Pilachowski (2013; magenta). A typical error bar is shown. See text for details.
Figure 4 has been corrected. In the previous version of this figure theoretical curves had a systematic offset in the $[\mathrm{F} / \mathrm{Fe}]$ ratio by a factor $\sim 0.2$ dex upwards. This has been corrected in the present version (below). This correction does not have any consequence on the conclusions of the paper. In fact, it shows even more clearly that, independently of the F yields from asymptotic giant branch (AGB) stars used (whether from Karakas 2010 or from Cristallo et al. 2015) in a simple Galactic chemical evolution model, it is impossible to account for the observed $[\mathrm{F} / \mathrm{Fe}] \mathrm{ra}-$ tios at the epoch of the solar system formation $([\mathrm{Fe} / \mathrm{H}] \approx 0.0)$. Therefore, other F sources are required. The caption of Fig. 4 remains the same.

\section{References}

Cristallo, S., Abia, C., Straniero, O., \& Piersanti, L. 2015, ApJ, 801, 53 Jönsson, H., Ryde, N., Harper, G. M., Richter, M. J., \& Hinkle, K. H. 2014, ApJ, 789, L41

Karakas, A. I. 2010, MNRAS, 403, 1413

Li, H. N., Ludwig, H.-G., Caffau, E., Christlieb, N., \& Zhao, G. 2013, ApJ, 765, 51

Maiorca, E., Uitenbroek, H., Uttenthaler, S., et al. 2014, ApJ, 788, 149

Nault, K. A., \& Pilachowski, C. A. 2013, AJ, 146, 153

Recio-Blanco, A., de Laverny, P., Worley, C., et al. 2012, A\&A, 538, A117 